\title{
Expression of a leptospiral leucine-rich repeat protein using a food-grade vector in Lactobacillus plantarum, as a strategy for vaccine delivery
}

\author{
Chattip Suphatpahirapol ${ }^{1,2} \cdot$ Thu-Ha Nguyen ${ }^{3}$. Yada Tansiri ${ }^{2,4} \cdot$ Yodying Yingchutrakul $^{5}$. Sittiruk Roytrakul ${ }^{1,5}$. \\ Supachai Nitipan ${ }^{6}$. Worawidh Wajjwalku ${ }^{1}$ - Dietmar Haltrich ${ }^{1,3} \cdot$ Siriwan Prapong ${ }^{1,2,4}$. Suttipun Keawsompong ${ }^{1,4,7}$ (i)
}

Received: 18 June 2019 / Accepted: 1 August 2019 / Published online: 8 August 2019

(c) King Abdulaziz City for Science and Technology 2019

\begin{abstract}
In this study, a first food-grade mucosal vaccine against leptospirosis was developed without the use of antibiotic resistance gene. This expression system is based on a food-grade host/vector system of Lactobacillus plantarum and a new vaccine candidate antigen, a leucine-rich repeat (LRR) protein of Leptospira borgpetersenii. The LRR of interest from serovar Sejroe is encoded by two overlapping genes and these genes were fused together by site-directed mutagenesis. The mutant gene thus obtained could be successfully expressed in this system as was shown by western blot analysis and liquid chromatography-mass spectrometry (LC-MS/MS) analysis. In addition, this analysis showed that the mutant LRR protein fused to a homologous signal peptide of $L$. plantarum could be exported to the cell surface as a result of the native LPXAG motif of the heterologous LRR protein, which presumably is responsible for anchoring the protein to the cell wall of L. plantarum. This new strategy could be an essential tool for further studies of leptospirosis mucosal vaccine delivery.
\end{abstract}

Keywords Lactobacillus plantarum $\cdot$ Leucine-rich repeat $\cdot$ Leptospiral protein $\cdot$ Food-grade expression system $\cdot$ Mucosal vaccine

Suttipun Keawsompong

fagisuk@ku.ac.th

1 Interdisciplinary Graduate Program in Genetic Engineering, Graduate School, Kasetsart University, Bangkok, Thailand

2 Department of Physiology, Faculty of Veterinary Medicine, Kasetsart University, Bangkok, Thailand

3 Department of Food Sciences and Technology, University of Natural Resources and Life Sciences, Vienna, Austria

4 Center for Advanced Studies for Agriculture and Food (CASAF), Institute for Advanced Studies, Kasetsart University, Bangkok, Thailand

5 National Center for Genetic Engineering and Biotechnology (BIOTEC), National Science and Technology Development Agency, Pathum Thani, Thailand

6 Department of Biology, Faculty of Sciences, Thaksin University, Phatthalung Campus, Phatthalung, Thailand

7 Department of Biotechnology, Faculty of Agro-Industry, Kasetsart University, Bangkok, Thailand

\section{Introduction}

One of the most wide spread zoonosis worldwide is leptospirosis caused by pathogenic Leptospira species. It is considered to be a re-emerging disease which impacts public health globally. Commercial leptospiral vaccines based on induction of antibodies against leptospiral lipopolysaccharide (LPS) can protect only against homologous or closely related serovars because of the diversity in the LPS carbohydrate structures of Leptospira, while more than 260 serovars of these pathogenic spirochetes were identified (Adler and de la Peña 2010; Brown et al. 2003). Therefore, several potential alternative vaccine candidates for heterologous protection have been studied. The outer membrane proteins are highly conserved across pathogenic Leptospira species (Branger et al. 2001); hence, many component proteins of the leptospiral outer membrane, including lipoproteins (such as LipL32 and LipL41), the leptospiral immunoglobulinlike proteins (such as LigA and LigB) and porins (such as OmpL1), have been studied as target antigens to induce protective immunity (Branger et al. 2001; Deveson Lucas et al. 
2011; Haake et al. 1999; Palaniappan et al. 2006; Silva et al. 2007).

An interesting target of antigen candidates is the leucinerich repeat (LRR). This structural motif is found in a number of proteins that appear to be involved in protein-protein interactions (Enkhbayar et al. 2004; Kobe and Kajava 2001). Previous studies have shown that the LRR protein has considerable potential as a vaccine candidate antigen. The LRR surface protein of Streptococcus agalactiae was tested as a vaccine antigen and shown to elicit protection against lethal challenge of virulent streptococci in mice (Seepersaud et al. 2005). An analysis of leptospiral LRR-containing genes has been performed, indicating that pathogenic Leptospira show a higher frequency of LRR genes than nonpathogenic species (Hniman and Prapong 2007). Furthermore, LRR proteins containing immunogenic epitopes were identified as well by Nitipan et al. (2013).

Since some pathogens, including Leptospires, enter the body via mucosal surfaces, using a mucosal vaccine to administer the antigen directly to the mucosal surface is the effective way to induce mucosal immunity instead of using conventional vaccine based on injection which lacks stimulation of local immunity. Gram-positive lactic acid bacteria (LAB) such as Lactobacillus species, which often carry the 'generally recognized as safe (GRAS)' status, are attractive for the development of mucosal vaccines. Several components of Lactobacillus spp., including their peptidoglycan, lipoteichoic acids, and bacterial oligodeoxynucleotides, elicit innate immune responses through interaction with molecular pattern recognition receptors of mammalian cells (Kim et al. 2006; Shimosato et al. 2005). Several strains of Lactobacillus have been studied for vaccine delivery, including Lactobacillus casei, Lactobacillus acidophilus, and Lactobacillus plantarum (del Rio et al. 2010; Mohamadzadeh et al. 2009; Shaw et al. 2000; Yigang and Yijing 2008).

In this study, we chose L. plantarum for reasons of survival in the gut, persistence in the host, and proven safety for consumption. Several strains of L. plantarum are marketed as probiotics, which confer various health benefits to humans (de Vries et al. 2006). L. plantarum is also considered a better agent for oral vaccination than $L$. casei or Lactococcus lactis (Cortes-Perez et al. 2007; Grangette et al. 2002; Shaw et al. 2000). Moreover, the complete genome sequence of $L$. plantarum WCFS1, a single colony isolate from $L$. plantarum NCIMB8826, which was originally isolated from human saliva, has been reported (Kleerebezem et al. 2003) and an inducible expression system for Lactobacillus hosts, namely the pSIP system, was developed and analyzed in some detail (Diep et al. 2009; Nguyen et al. 2011a, b; Sørvig et al. 2003, 2005). A food-grade system for the pSIP expression vector series was recently developed (Nguyen et al. 2011a, b). To construct a food-grade complementation-based system, the erythromycin antibiotic resistance gene ( $\mathrm{erm})$ was replaced by the alanine racemase gene $(a l r)$ as selection marker complementing the corresponding gene deletion in the chromosome of the host $L$. plantarum WCFS1. Thus, it is suitable for food-associated application.

The objective of the present study was to develop an expression system for leptospiral genes in nonpathogenic LAB using a food-grade system as a new strategy for leptospirosis vaccine delivery. Here, we describe the construction of recombinant $L$. plantarum expressing a leptospiral LRR antigen. Furthermore, the food-grade pSIP system containing an L. plantarum homologous signal peptide was also developed to direct the target antigen to the cell surface as well. These recombinant bacteria carrying the LRR antigen would be the first mucosal vaccine development against leptospirosis which is not a conventional needle-based vaccine and does not contain any antibiotic resistance gene.

\section{Methods}

\section{Bacterial strains, plasmids and media}

The bacterial strains and plasmids used in this study are listed in Table 1. E. coli MB2159 (D-alanine auxotroph) was used as cloning hosts, whereas L. plantarum TLG02 (WCFS1 derivative, D-alanine auxotroph) was used as hosts for expression. E. coli was grown in Luria-Bertani medium at $37{ }^{\circ} \mathrm{C}$ with shaking. L. plantarum was grown in deMan-Rogosa-Sharpe (MRS) medium at $30{ }^{\circ} \mathrm{C}$ in tightly capped flasks and without agitation, D-alanine (Sigma, St. Louis, MO, USA) was added at a final concentration of $200 \mu \mathrm{g} / \mathrm{mL}$ for negative control (L. plantarum TLG02 without vector).

\section{Construction of leptospiral LRR protein expression vectors and transformation}

Primers used in this study were purchased from VBC-Biotech Service, Austria (Table 2). To construct pSIP vectors containing the Sejroe LRR genes for $L$. plantarum expression, the plasmid KU_pET160_R21F_2012, carrying LRR of Leptospira borgpetersenii serovar Sejroe (NCBI accession number JN627495), was used as a template to amplify two overlapping genes, $K U \_S e j \_R 21 N \_2012$ gene (NCBI accession number JN627491) and $K U \_S e j \_R 21 C \_2012$ gene (NCBI accession number JN627492). For full-length LRR protein expression, site-directed mutagenesis of these LRR overlapping genes was performed from plasmid KU_pET160_R21F_2012 using the overlap extension PCR method (Ho et al. 1989). The R21_NcoI and R21N_Mut primers were used to create the $\mathrm{N}$-terminal fragment $\left(K U_{-}\right.$ Sej_R21N_2012), while the R21C_Mut and R21C_EcoRI_ St primers were used to create the C-terminal fragment 
Table 1 Bacterial strains and plasmids used in this study

\begin{tabular}{|c|c|c|}
\hline Strains and plasmids & Characteristics $^{\mathrm{a}}$ & Source/references \\
\hline \multicolumn{3}{|l|}{ Strains } \\
\hline E. coli $\mathrm{MB} 2159$ & Host strain for cloning, D-alanine auxotroph & Nguyen et al. (2011a, b) \\
\hline L. plantarum TLG02 & Host strain for expression, WCFS 1 derivative, D-alanine auxotroph & Nguyen et al. (2011a, b) \\
\hline \multicolumn{3}{|l|}{ Plasmids } \\
\hline KU_pET160_R21F_2012 & $\begin{array}{l}\text { pET160/GW/D-TOPO derivative, containing } K U \_S e j \_R 21 F \_2012 \text { from Leptospira } \\
\text { borgpetersenii serovar Sejroe, } \mathrm{Am}^{\mathrm{r}}\end{array}$ & Nitipan (2013) \\
\hline pSIP609 & pSIP409 derivative, $e r m$ replaced by $a l r$ & Nguyen et al. (2011a, b) \\
\hline pSIP609-KU_R21M_2012 & pSIP609 derivative, gusA replaced by $K U \_S e j \_R 21 M \_2012$ & This work \\
\hline pLp_2145sAmy & pSIP401 derivative, $\mathrm{P}_{s p p A}, s p_{L p_{-} 2145}$ fused to amyA, $\mathrm{Em}^{\mathrm{r}}$ & Mathiesen et al. (2009) \\
\hline pLp_2145sAlr-KU_R21M_2012 & $\begin{array}{l}\text { pLp_2145sAmy derivative, } e r m \text { replaced by alr, amyA replaced by } K U \_S e j \_ \\
R 21 M \_2012\end{array}$ & This work \\
\hline
\end{tabular}

${ }^{a}$ alr alanine racemase encoding gene, erm erythromycin resistance gene, amyA alpha-amylase encoding gene, $A m^{r}, E m^{r}$ ampicillin and erythromycin resistance, respectively

Table 2 Primers used in this study

\begin{tabular}{ll}
\hline Primers & Sequences $\left(5^{\prime} \rightarrow 3^{\prime}\right)^{\mathrm{a}}$ \\
\hline R21_NcoI & GGCGCCATGGGAAAAAATGAATG $($ NcoI $)$ \\
R21N_Mut & GGAAGTTTTGACAAAAGACCGAGATC \\
R21C_Mut & GATCTCGGTCTTTTGTCAAAACTTCC \\
R21C_EcoRI_St & CCGGGAATTCTTAAAATGAGATTTTCAC \\
& ATCG $($ EcoRI $)$ \\
Fsig_PstI & TATGCTGCAGCACGCCAAATGATC $($ Pst I $)$ \\
R2145_SalI & GTAAGTCGACGGCGCTAGCAT $($ SalI $)$ \\
R21F_SalI & CGTTGTCGACCAGGGAAAAAATGAA (SalI) \\
\hline
\end{tabular}

${ }^{\mathrm{a}}$ The restriction sites are underlined

(KU_Sej_R21C_2012) during the first round of PCR. These fragments were mixed for the second round of PCR using primers R21_NcoI and R21C_EcoRI_St to create the mutant LRR gene, named $K U \_S e j \_R 21 M \_2012$ (Fig. 1a). The final fragment was digested with $N c o I$ and EcoRI, and ligated into the $\sim 6 \mathrm{~kb} \mathrm{NcoI-EcoRI} \mathrm{digested} \mathrm{fragment} \mathrm{of} \mathrm{plasmid}$ pSIP609, yielding the pSIP609-KU_R21M_2012 plasmid for expression in L. plantarum (Fig. 1b).

To construct expression vectors carrying a homologous signal peptide of $L$. plantarum, a fragment containing the $L$. plantarum signal peptide $L p \_2145$ fused to the promoter $\mathrm{P}_{s p p A}$ was amplified from plasmid pLp_2145sAmy using the Fsig_PstI and R2145_SalI primers, and digested with PstI and SalI. pSIP609-KU_R21M_2012 was used as a template for PCR amplification of the $K U_{-} S e j \_R 21 M \_2012$ fragment using the R21F_SalI and R21C_EcoRI_St primers. The gene fragment was digested with SalI and EcoRI. The signal peptide and gene fragments were then ligated into a $~ 5.5 \mathrm{~kb} P s t \mathrm{I}-E c o$ RI digested fragment of pSIP609, resulting in pLp_2145sAlr-KU_R21M_2012 (Fig. 1b). The correct nucleotide sequence of all inserts was verified by DNA sequencing. All pSIP vectors were constructed in E. coli
MB2159 before electroporation into L. plantarum TLG02 according to the method described previously (Josson et al. 1989).

The nucleotide sequence of $K U \_S e j \_R 21 M \_2012$ was translated into the amino acid sequence using a web-based tool, ExPASy Proteomics Server (http://web.expasy.org/ translate/) and was compared with the LRR of Lp. borgpetersenii serovar Hardjo-bovis strain JB197 using a webbased tool, Clustal Omega (https://www.ebi.ac.uk/Tools/ $\mathrm{msa} /$ clustalo/). The potential T cell and B cell epitopes were predicted by MHCPred (https://www.jener.ac.uk/MHCPr ed) and antigenic (http://emboss.bioinformatics.nl/cgi-bin/ emboss/antigenic) programs, respectively.

\section{Expression of mutant LRR protein in L. plantarum}

Overnight culture of $L$. plantarum carrying the respective plasmids was diluted in fresh prewarmed MRS medium to an $\mathrm{OD}_{600}$ of $\sim 0.1$. Then, $50 \mathrm{~mL}$ of bacterial culture was incubated at $30{ }^{\circ} \mathrm{C}$ without shaking until the absorbance reached an $\mathrm{OD}_{600}$ of $\sim 0.3$. The cells were induced by adding $50 \mathrm{ng} /$ $\mathrm{mL}$ of the 19-residue peptide pheromone (IP) IP-673, the specific inducer for an inducible promoter $\mathrm{P}_{s p p A}$ of Lactobacillus sp., and harvested at $\mathrm{OD}_{600}$ of $\sim 6-7$ by centrifugation at $3200 \mathrm{~g}$ for $15 \mathrm{~min}$ at $4{ }^{\circ} \mathrm{C}$.

\section{Cell extract preparation and western blot analysis}

The supernatant was collected and concentrated 50 times to examine secreted proteins by centrifugal filters. The cell pellet was washed once with LEW buffer ( $50 \mathrm{mM}$ of sodium phosphate, $300 \mathrm{mM}$ of sodium chloride, $\mathrm{pH} 8.0$ ) and resuspended in $10 \mathrm{~mL}$ of the same buffer. The cells were disrupted by glass beads using a Precellys 24 glass bead mill (PEQLAB Biotechnologie GmbH, Germany). 
a

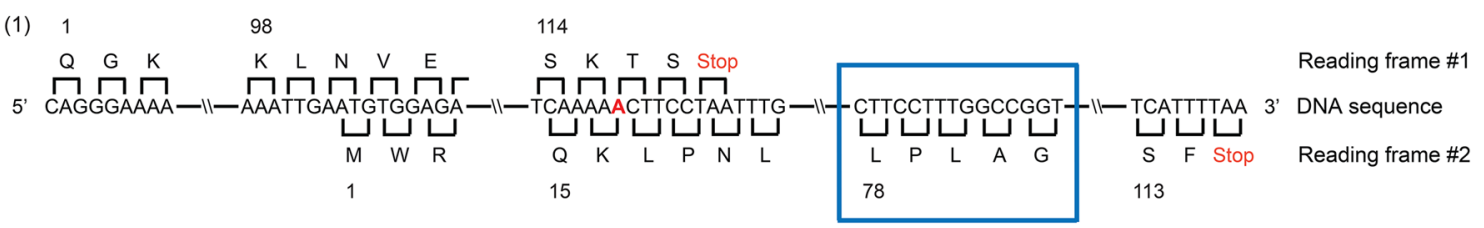

KU_Sej_R21N_2012

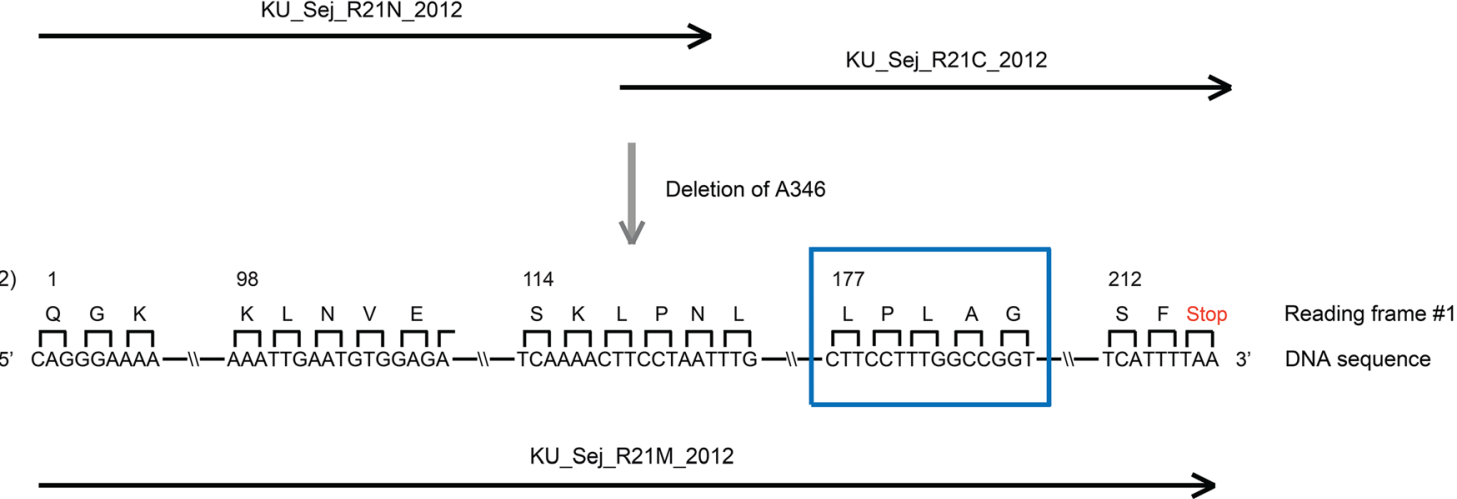

b

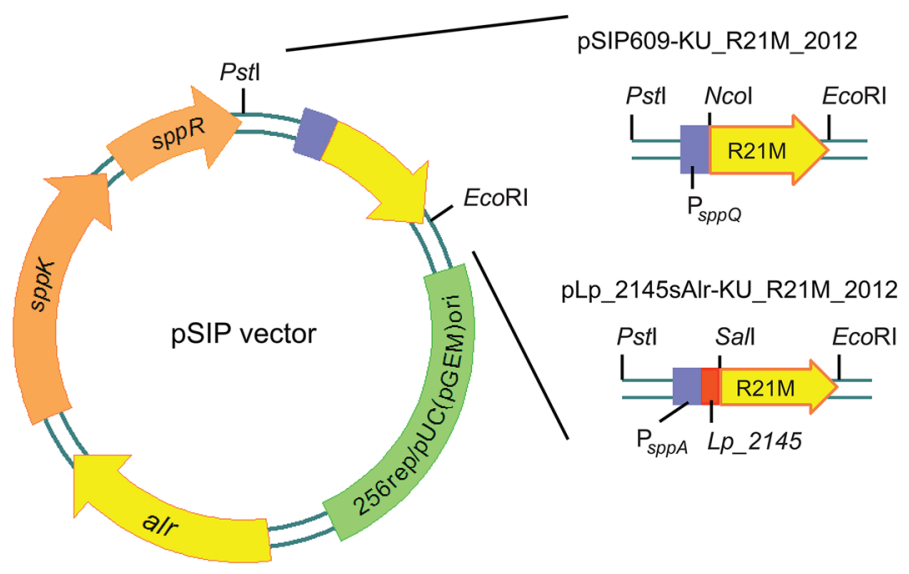

Fig. 1 Schematic diagram of DNA sequences (a) and expression vectors (b). DNA sequences and reading frames of the Lp. borgpetersenii serovar Sejroe LRR genes before (1) and after (2) a single base-pair deletion at A346 (marked by bold red A). The LPXTG motif (LPXAG) is marked by the blue box. The black arrows represent the gene fragments. Expression vectors for the full-length LRR protein, using the alanine racemase (alr) gene as selection marker.

For total protein extraction, $1 \mathrm{~mL}$ of the whole cell lysate was heated with $1 \mathrm{~mL}$ of $2 \times$ SDS-PAGE sample buffer (0.2 M dithiothreitol, 4\% SDS, $0.1 \mathrm{M}$ Tris, $\mathrm{pH} 6.8,20 \%$ glycerol and $0.2 \%$ bromophenyl blue) at $95{ }^{\circ} \mathrm{C}$ for $5 \mathrm{~min}$, and cell debris was removed by centrifugation at $16,000 \mathrm{~g}$ for 5 min at $4{ }^{\circ} \mathrm{C}$ to obtain the total protein extract.

To separate insoluble proteins, cytosolic proteins and surface proteins, the remaining whole cell lysate was centrifuged at $16,000 \mathrm{~g}$ for $5 \mathrm{~min}$ at $4{ }^{\circ} \mathrm{C}$. The pellet was collected for insoluble proteins extraction, while supernatant was kept for soluble protein extraction. The pellet for insoluble
SppK and $\operatorname{sppR}$, histidine kinase and response regulator, respectively; 256rep/pUC(pGEM)ori, replication determinant. The structural gene (KU_Sej_R21M_2012 gene of leptospiral LRR, indicated by 'R21M') is fused to the inducible promoter $\mathrm{P}_{s p p Q}$ in the pSIP609-KU_ $\mathrm{R} 21 \mathrm{M} \_2012$ vector $(6654 \mathrm{bp})$ and fused to $\mathrm{P}_{s p p A}$ with a signal peptide of L. plantarum, Lp_2145, in the pLp_2145sAlr-KU_R21M_2012 vector $(6755 \mathrm{bp})$

proteins extraction was washed once with LEW buffer and resuspended in $2 \mathrm{~mL}$ denaturing solubilization buffer (50 mM NaH $\mathrm{PO}_{4}, 300 \mathrm{mM} \mathrm{NaCl}, \mathrm{pH} 8.0,8 \mathrm{M}$ urea, $\mathrm{pH}$ 8.0) per $\mathrm{g}$ wet cells, stirred on ice for $60 \mathrm{~min}$ and centrifuged at $16,000 \mathrm{~g}$ for $5 \mathrm{~min}$ at $20^{\circ} \mathrm{C}$ to remove cell debris. The supernatant was kept at $4{ }^{\circ} \mathrm{C}$ as an insoluble protein extract.

Then, the soluble protein fraction was centrifuged at $80,000 \mathrm{~g}$ for $90 \mathrm{~min}$ at $4{ }^{\circ} \mathrm{C}$ using a Beckman L-70 Ultracentrifuge to separate cytosolic proteins and surface proteins. The supernatant was collected as cytosolic proteins extract, whereas the pellet was resuspended in LEW buffer 
and collected as surface protein extract. Protein concentration was determined using the Bradford protein assay with bovine serum albumin (BSA) as standard.

Each protein extract was mixed with SDS-PAGE sample buffer and denatured at $95{ }^{\circ} \mathrm{C}$ for $5 \mathrm{~min}$. Twelve micrograms of the surface proteins and $15 \mu \mathrm{g}$ of the proteins from other extracts were applied to the individual lanes of SDS-PAGE gels. SDS-PAGE was carried out on $14 \%$ acrylamide gels and the protein bands were transferred electrophoretically onto nitrocellulose membranes for western blot using a Bio-Rad electrophoresis unit. Rabbit hyperimmune serum against the KU_Sej_LRR_2012N protein (1:5000 dilution) was used as primary antibody for the blot. The secondary antibody was a peroxidase-conjugated goat-anti-rabbit IgG (Jackson ImmunoResearch Laboratories, Inc.) at a dilution of 1:10,000. Blot was developed using the Bio-Rad Clarity $^{\mathrm{TM}}$ western ECL substrate kit, according to the manufacturer's instructions.

To acquire rabbit hyperimmune serum as primary antibody, the gel fragment approach was used for immunization (Harlow and Lane 1988). Briefly, the gel slice containing the KU_Sej_LRR_2012N protein from the previous study (Nitipan 2013) was rinsed in deionized water for a few minutes and was lyophilized for $48 \mathrm{~h}$. Then, the dried gel slice was moved to a mortar and was ground into a fine powder. A volume of PBS equal to one-half of the original gel volume was added and incubated at room temperature for $1 \mathrm{~h}$. Complete Freund's adjuvant (FCA) and incomplete Freund's adjuvant (FIA) were mixed with an equal volume of the protein antigen for the primary injection and all boosts, respectively. A New Zealand White rabbit received four $1.2 \mathrm{~mL}$ doses of the sample containing $0.6 \mathrm{mg}$ protein antigen on days $0,14,176$ and 363 . Rabbit serum was collected twice a month. Animal experiments were approved by the Kasetsart University Institutional Animal Care and Use Committee (Kasetsart University-IACUC). To calculate antibody titer, the serum sample was diluted in serial ratios and tested for the presence of detectable levels of antibody. The titer value was indicative of the last dilution in which the antibody was detected. The serum with a high titer of more than 12,800 was collected and absorbed with $E$. coli BL21 (DE3) acetone powder before use as primary antibody.

\section{Flow cytometric analysis}

A million cells of L. plantarum carrying pLp_2145sAlrKU_R21M_2012 were incubated with rabbit hyperimmune serum against KU_Sej_LRR_2012N protein at a dilution of 1:600 and were stained with fluorescein isothiocyanate (FITC)-conjugated goat anti-rabbit IgG (AbD Serotec) at a dilution of 1:600 as secondary antibody. At least 100,000 events were analyzed using the CytoFLEX flow cytometer running with CytExpert software (Beckman Coulter).
Negative controls were L. plantarum cells harboring plasmid and without plasmid, which were stained with only FITC-conjugated goat anti-rabbit IgG and with both rabbit hyperimmune serum and FITC-conjugated goat anti-rabbit IgG, respectively. Fluorescent signals were represented by histograms.

\section{Liquid chromatography-mass spectrometry analysis (LC-MS/MS)}

The gel slices containing the KU_Sej_LRR_2012M protein from gel electrophoresis were subjected to in-gel digestion and dehydrated twice by $100 \%$ acetonitrile (ACN). Sulfhydryl bonds were reduced by $10 \mathrm{mM}$ tributylphosphine (TBP) in $10 \mathrm{mM}$ ammonium bicarbonate for $1 \mathrm{~h}$ at $60^{\circ} \mathrm{C}$. Then, sulfhydryl groups were alkylated with $100 \mathrm{mM}$ iodoacetamide (IAA) in $10 \mathrm{mM}$ ammonium bicarbonate and left in the dark for $45 \mathrm{~min}$ at room temperature. The gel slices were dehydrated twice again and digested by adding 100-200 ng of sequencing grade trypsin. The gel slices were incubated for $1 \mathrm{~h}$ at $4{ }^{\circ} \mathrm{C}$ followed by $37{ }^{\circ} \mathrm{C}$ overnight. The digested peptide fragments were extracted by adding $30 \mu \mathrm{L}$ of $50 \%$ ACN in $0.1 \%$ formic acid and incubated for $10 \mathrm{~min}$ at room temperature with shaking. The peptide fragments were dried at $44{ }^{\circ} \mathrm{C}$ and protonated with $0.1 \%$ formic acid before injection into a NanoAcquity system. The peptide fragments were analyzed using an Ultimate3000 Nano/Capillary LC System (Dionex, UK) coupled to a hybrid quadrupole Q-Tof impact II ${ }^{\mathrm{TM}}$ (Bruker Daltonics $\mathrm{GmbH}$, Germany) with a nano-captive spray ion source. Sample $(500 \mathrm{~nL})$ was loaded into the trapping column (Thermo Scientific), using full loop injection, and resolved on an analytical column (PepSwift Monolithic Nano Column) at a column temperature of $60{ }^{\circ} \mathrm{C}$. A linear gradient method was used to elute peptides into the mass spectrometer at a constant flow rate of $1 \mu \mathrm{L} / \mathrm{min}$ of the mobile phases A (0.1\% formic acid in water) and B $(0.1 \%$ formic acid in $80 \% \mathrm{ACN})$. Electrospray ionization was carried out at $1.6 \mathrm{kV}$ using the CaptiveSpray. Mass spectra were acquired in the positive-ion mode over the range $\mathrm{m} / \mathrm{z}$ 150-2200 (Compass 1.9 for otofSeries software, Bruker Daltonics) and the MS/MS data were analyzed using MASCOT software (Matrix Science, UK) with the target protein database. Ion score was $-10 \times \log (P)$, where $P$ was the probability that the observed match was a random event. Protein score was derived from ion scores as a nonprobabilistic basis for ranking protein hits. The peptide sequences from mass spectrometry were aligned with the KU_Sej_LRR_2012M sequence using a web-based tool, Clustal Omega (https://www.ebi.ac.uk/Tools/msa/clust alo/). 


\section{Results}

\section{Leptospiral LRR gene expression in L. plantarum with the food-grade pSIP system after fusion of the two overlapping genes}

Since the LRR protein of interest of Lp. borgpetersenii serovar Sejroe is encoded by two overlapping genes, $K U_{-}$ Sej_R21N_2012 (NCBI accession number JN627491) and $K U \_S e j \_R 21 C \_2012$ (NCBI accession number JN627492), we decided to fuse the two overlapping genes of the Sejroe LRR for full-length LRR protein expression. PCR sitedirected mutagenesis was performed for a single base-pair deletion (A346), resulting in $K U \_S e j \_R 21 M \_2012$, which consists of both the $K U \_S e j \_R 21 N \_2012$ and the $K U \_S e j \_$ $R 21 C \_2012$ genes in frame (Fig. 1a). The recombinant pSIP vector containing $K U \_S e j \_R 21 M \_2012$ was constructed and named pSIP609-KU_R21M_2012 (Fig. 1b).

From in silico identification, an amino acid sequence of the mutant LRR protein of Sejroe was compared to that of the LRR of serovar Hardjo-bovis strain JB197 (LBJ_2012 gene, NCBI accession number ABJ76523) and showed $85 \%$ similarity (Fig. 2). This fused protein contained sequence encoding each of the four target epitopes for $\mathrm{T}$ cell and B cell. In cell-mediated immunity (CMI) system, $\mathrm{T}$ cell activation requires the antigen to bind to major histocompatibility complex (MHC) molecules. According to this prediction, the result showed epitopes for HLAA0101, -A3101 and -A0203 alleles as MHC class I and for HLA-DRB0401 allele as MHC class II. For humoral immunity (HI), B cell epitope is the antigen portion recognized by $\mathrm{B}$ cells and binds to the immunoglobulin. The scores of predicted epitopes were 1.121, 1.137, 1.145 and 1.140 , respectively (Fig. 2). The epitopes having more than 1.0 are potentially antigenic. These conserved epitopes were also found in serovar Ballum, Javanica and Mini (Nitipan 2013).

L. plantarum TLG02 was used as the host of the vector and analyzed after induction with IP-673. The formation of the mutant LRR protein in L. plantarum could be detected by western blot analysis (Fig. 3a). The target protein was apparently expressed in both soluble and insoluble forms.

\section{LRR protein expression with an L. plantarum signal peptide}

To develop an expression system for cell surface display of the LRR protein, we tested whether it is possible to secrete the recombinant mutated LRR protein. $K U \_S e j \_R 21 M \_2012$ was first fused to the signal peptide Lp_2145 of L. plantarum before ligation into the pSIP vector for $L$. plantarum expression, resulting in pLp_2145sAlr-KU_R21M_2012 (Fig. 1b). Western blot analysis of different samples obtained from induced $L$. plantarum cells showed target protein bands in several fractions, including insoluble protein, cytoplasmic protein and surface protein fractions (Fig. 3a). No mutant LRR protein was found in the culture supernatant (exhausted media).

\section{Analysis of protein localization}

Expression of the $K U \_S e j \_R 21 M \_2012$ gene as a surface-anchored protein on $L$. plantarum carrying

KU_Sej_LRR_2012M
ABJ76523.1_LRR
KU_Sej_LRR_2012M
ABJ76523.1_LRR
KU_Sej_LRR_2012M
ABJ76523.1_LRR
KU_Sej_LRR_2012M
ABJ76523.1_LRR
KU_Sej_LRR_2012M
ABJ76523.1_LRR

Fig. 2 Amino acid sequence alignment of the KU_Sej_LRR_2012M sequence with the LRR of Lp. borgpetersenii serovar Hardjo-bovis strain JB197. The LPXTG motif (LPXAG) is marked by the yellow

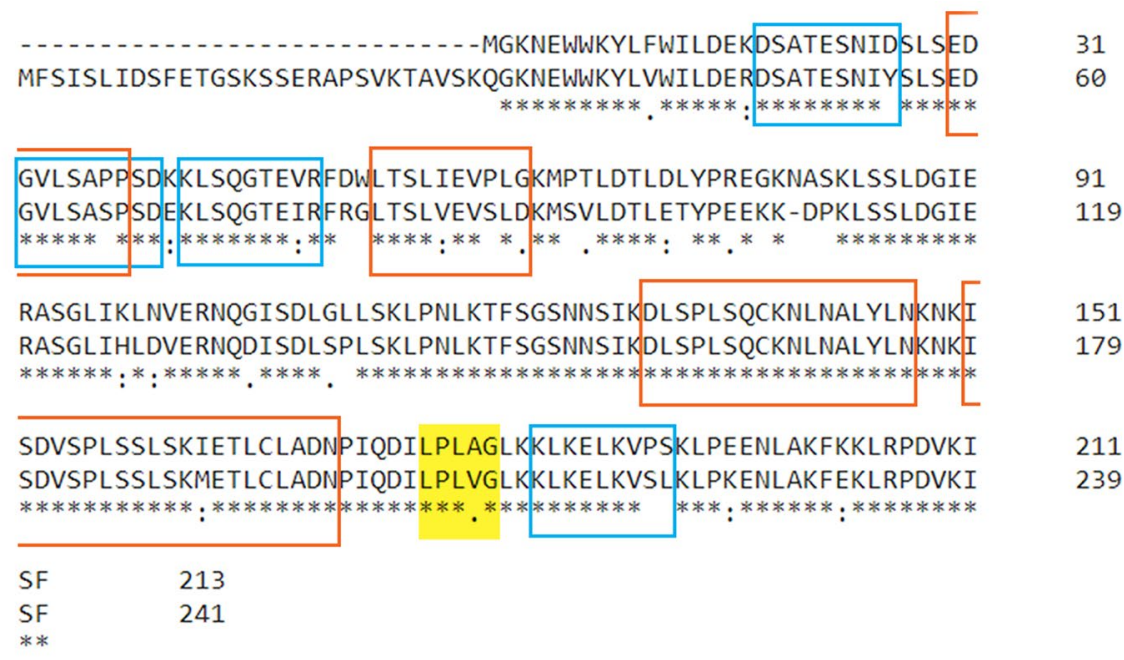

box. The KU_Sej_LRR_2012M sequence encoding target epitopes for MHC HLA alleles and B cell is marked by blue and red boxes, respectively 
a

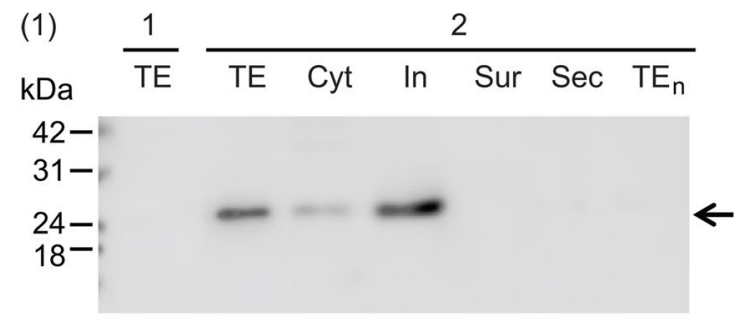

(2)

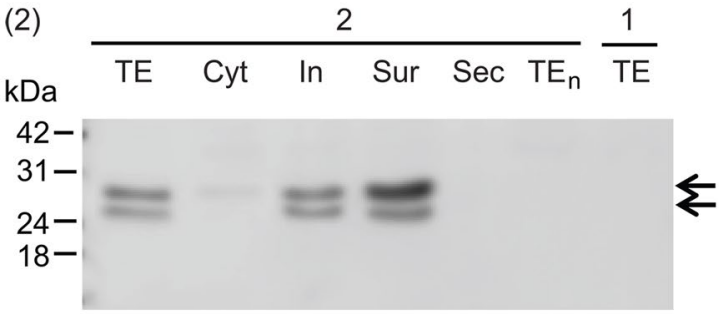

b

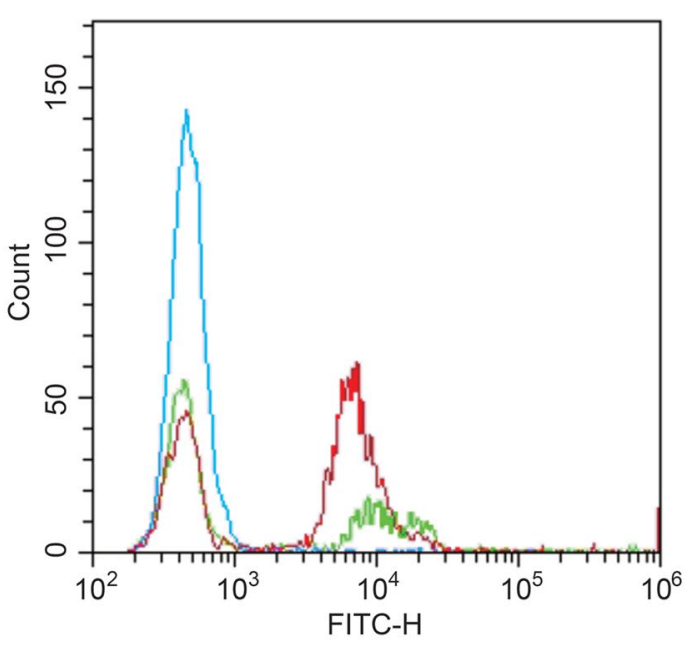

of the mutated LRR protein fused to the Lp_2145 signal peptide, with $27.5 \mathrm{kDa}$ and $24 \mathrm{kDa}$ for the unprocessed and processed proteins, respectively. Lactobacilli were gated by forward and side scatter and surface-anchored expression of the LRR protein was analyzed as histogram. The fluorescent signals from $L$. plantarum harboring the pLp_2145sAlr-KU_R21M_2012 plasmid and stained with both antibodies, rabbit hyperimmune serum against KU_Sej_LRR_2012N protein and FITC-conjugated goat anti-rabbit IgG (red line), L. plantarum harboring the pLp_2145sAlr-KU_R21M_2012 plasmid and stained with only FITC-conjugated goat anti-rabbit IgG (blue line), and $L$. plantarum without plasmid with both antibodies (green line) are shown

our result showed a Mascot protein score of 1466, it is very likely that the expressed protein is the KU_Sej_LRR_2012M protein.

Furthermore, 11 peptide matches were found (Table 3) showing $65.7 \%$ of sequence coverage (Fig. 4b). This analysis corroborates that the expressed protein recognized by the rabbit hyperimmune serum is indeed the LRR protein.

\section{Discussion}

In this study, we expressed the leptospiral LRR in L. plantarum aiming at the development of a mucosal vaccine candidate against leptospirosis. The LRR of Lp. borgpetersenii serovar Sejroe is of interest for vaccine candidates as it would be a surface protein and an immunoreactive antigen. The LRR from one of these two overlapping genes, $K U \_S e j \_R 21 N \_2012$ (NCBI accession number JN627491), showed immunoreactivity to rabbit hyperimmune serum anti-Sejroe, Lp. borgpetersenii and anti-Ballico, Lp. interrogans antibodies provided by the National Institute of Animal Health, Department of Livestock Development, Thailand, whereas the latter, the LRR from $K U \_S e j \_R 21 C \_2012$ larity at $95 \%$ confidence level is above a score of 90 . Since

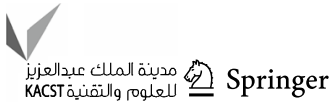




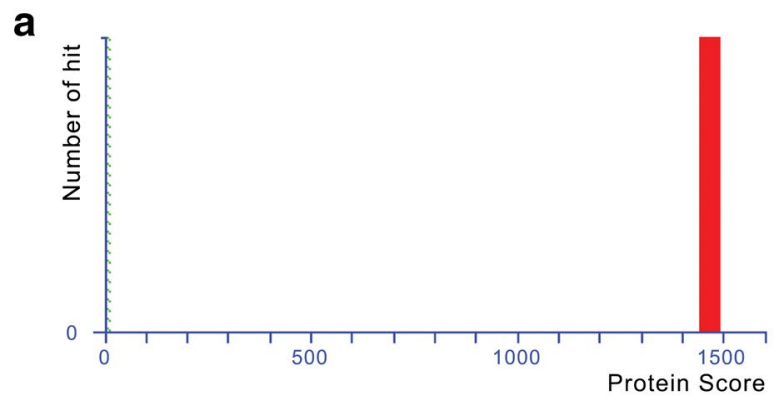

b

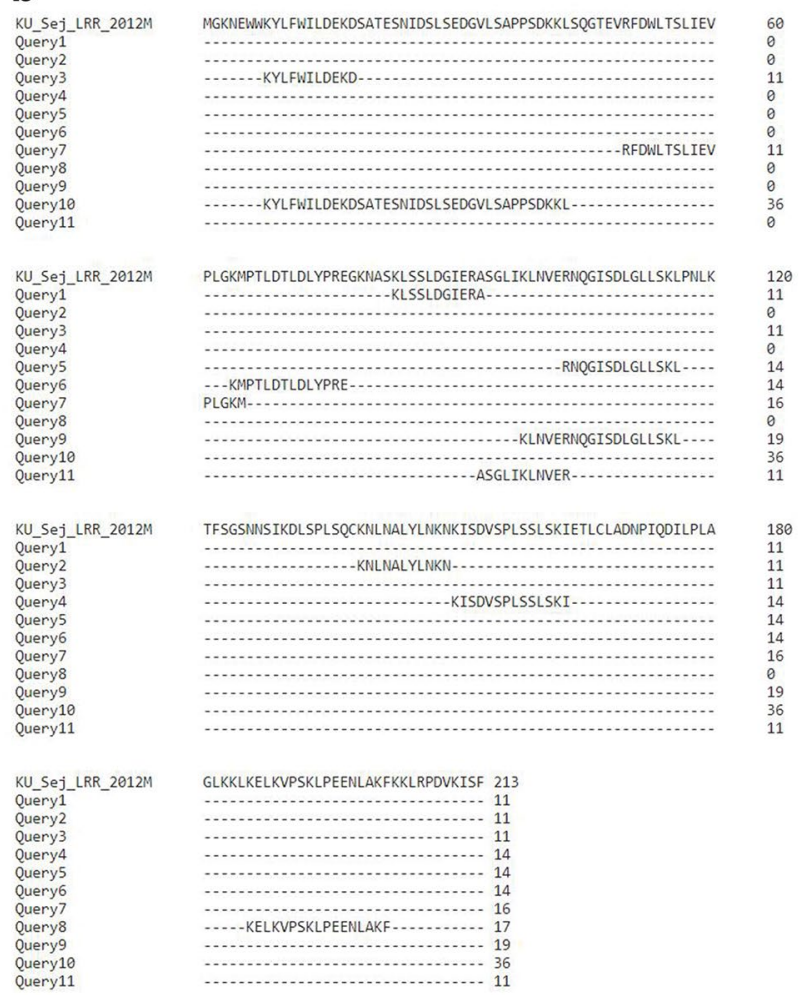

Fig. 4 Mascot score histogram (a) and alignment of peptide sequences (b) from LC-MS/MS analysis of the protein band at $23.5 \mathrm{kDa}$ expected as the KU_Sej_LRR_2012M protein using in-gel trypsin digestion method

(NCBI accession number JN627492), was detected with rabbit hyperimmune serum anti-Javanica and anti-Ballico, Lp. borgpetersenii (Nitipan 2013). Since we would like to express both of them in lactic acid bacteria, similar observations have been made for other heteromeric proteins such as for the $\beta$-galactosidase from Lactobacillus sakei. This enzyme is encoded by two overlapping genes (LacLM). The active $\beta$-galactosidase could not be produced when only expressing one of the two subunits (Obst et al. 1995). Moreover, the full-length LRR protein of another serovar, Lp. borgpetersenii serovar Hardjo-bovis strain JBL197, is encoded by only one gene (LBJ_2012 gene, NCBI accession number ABJ76523). The LRR gene sequence of this serovar is quite similar to that of serovar Sejroe (91.4\%),
Table 3 Peptide matches from the protein band at $23.5 \mathrm{kDa}$ identified by mass spectrometry

\begin{tabular}{lll}
\hline Peptide sequences from Mascot & Ion score $^{\text {a }}$ & $\begin{array}{l}\text { Num- } \\
\text { ber of } \\
\text { queries }\end{array}$ \\
\hline KLSSLDGIERA & $54-69$ & 3 \\
KNLNALYLNKN & 37 & 2 \\
KYLFWILDEKD & 38 & 2 \\
KISDVSPLSSLSKI & $25-26$ & 2 \\
RNQGISDLGLLSKL & $17-54$ & 9 \\
KMPTLDTLDLYPRE & $77-88$ & 6 \\
RFDWLTSLIEVPLGKM & $53-102$ & 6 \\
KELKVPSKLPEENLAKF & 42 & 1 \\
KLNVERNQGISDLGLLSKL & $31-36$ & 2 \\
KYLFWILDEKDSATESNID- & 83 & 1 \\
$\quad$ SLSEDGVLSAPPSDKKL & & 1 \\
ASGLIKLNVER & $5^{\mathrm{b}}$ & \\
\hline${ }^{a}$ Individual ion scores $>13$ indicate identity or extensive homology \\
$(P<0.05)$ \\
bPeptide match is not assigned to protein hits
\end{tabular}

except that the complete LBJ_2012 gene is in one reading frame and does not separate into two overlapping genes. In other serovars, LRR amino acid sequence of serovar Pomona (LEP1GSC133_2670, NCBI accession number EMO64909) is encoded by one gene same as serovar Hardjo-bovis (98\% peptide identity with Hardjo-bovis). However, two overlapping genes of LRR were also found in serovar Ballum and Javanica (Nitipan 2013). Based on the information given above, we decided to fuse the two overlapping LRR genes, $K U \_S e j \_R 21 N \_2012$ and $K U \_S e j \_R 21 C \_2012$, obtaining the mutated gene $K U \_S e j \_R 21 M \_2012$.

The corresponding protein was detected via western blot analysis, indicating that this mutant LRR protein derived from the fused genes was expressed as both soluble and insoluble forms in L. plantarum. The insoluble LRR protein may cause a problem for in vivo experiments due to the pathogenic misfolded proteins (for example, amyloid- $\beta$ plaques). However, in some cases, the insoluble proteins do not cause disease (Trimpin and Brizzard 2009). Further experiments on its property would be necessary. As an inhouse specific antibody was used in this study, it seemed necessary to further confirm that the expressed protein was indeed the target LRR protein by determination of the amino acid sequence of the expressed protein using LC-MS/MS analysis. Identified peptides were accepted with a total ion score of over 30 and more than five peptide matches. The data obtained by LC-MS/MS method corroborate the result obtained from western blot analysis. Therefore, the mutated gene $K U \_S e j \_R 21 M \_2012$ was successfully expressed in the lactobacillal host and the in-house rabbit hyperimmune serum can be used to recognize the target protein. 
Previous studies on vaccine development reported that an antigen displayed on the surface of LAB can induce mucosal and systemic immune responses (del Rio et al. 2008; Maassen et al. 1999; Medaglini et al. 1995). In addition, membrane-associated leptospiral antigens could induce significant protection in hamsters challenged with Leptospira, while non-membrane-associated forms of them were not protective. This suggests that the system, in which the antigens associate with the cell surface, is important for the induction of a protective immune response (Haake et al. 1999). Our LRR protein contains the pentapeptide LPXAG, which has a similar function as the LPXTG motif, a cell wall anchor recognized by sortase (Roche et al. 2003). Various signal peptides of $L$. plantarum WCFS1 have been analyzed with respect to protein secretion and were shown to export different heterologous proteins to the cell wall or the culture medium (Fredriksen et al. 2010; Mathiesen et al. 2008, 2009). Lp_2145 is one of the signal peptides of $L$. plantarum that was found to be very effective in these previous studies and we fused this signal peptide to the $\mathrm{N}$-terminus of the mutant LRR gene. After expression the target protein was, however, not detected as a secreted soluble protein in the culture supernatant, but we found a strong signal for the LRR protein associated with the cell surface fraction of $L$. plantarum. Presumably, the native LPXAG motif in KU_Sej_LRR_2012M resulted in anchoring of the recombinant protein on the cell wall of the expression host. Our data thus support the finding that a homologous signal peptide can be useful in food-grade systems to direct a heterologous protein to the Sec translocation machinery. Normally, Sec machinery recognizes the signal peptide on the target protein, cleaves the signal peptide off and exports the secreted protein outside the cell. In this step, our protein was not secreted due to the presence of a hydrophobic region terminated by a positively charged tail at the $\mathrm{C}$-terminus. As sortase anchoring system in Gram-positive bacteria, the protein remains embedded in the membrane because of its tail. Then, the enzyme cleaves the target protein between the glycine and alanine residues of LPXAG. The residue links to the peptide cross bridge in the peptidoglycan of the cell surface. Therefore, the LRR protein from Lp. borgpetersenii, Gram-negative bacteria, could be anchored to the cell surface of $L$. plantarum by its own, native sequence LPXAG.

Normally, leptospiral vaccine has been developed based on conventional injection so far, which can induce only systemic immunity and lack stimulation of local immunity. The effective way to induce both local and systemic immunity is the mucosal vaccine system which recombinant bacteria carrying the antigen are administered directly to the body via needle-free oral injection. This study showed the first construction of mucosal vaccine against leptospirosis. In addition, Lactobacillus spp. has been used for mucosal vaccines against several diseases. However, most of them used an antibiotic resistant gene as selection marker of the vector. In this study, we used the food-grade complementationbased system instead of antibiotics to prevent the release of antibiotic resistant bacteria to the environment. Using the pSIP expression vectors with the novel mutant LRR gene described here, the protein of interest could be produced and anchored to the surface of recombinant $L$. plantarum by the anchoring domain of leptospiral native motif. The new method of leptospiral antigen production using the foodgrade expression system of LAB could be a first and important step in the development of a mucosal vaccine against leptospirosis.

Acknowledgements This work was supported by Kasetsart University [grant \# 5410920] for CS and the OeAD-Austrian Agency for International Cooperation in Education \& Research [ICM-2012-00599] for CS. THN acknowledges the support from the Austrian Science Fund [FWF Project V457-B22], and THN and DH thank ASEA-Uninet for financial support. YT acknowledges the Center for Advanced Studies for Agriculture and Food (CASAF), Kasetsart University [grant \# CASAF PD008] for financial support. We are very grateful to Dr. Geir Mathiesen for kindly providing plasmid pLp_2145sAmy, Dr. TienThanh Nguyen for more information on plasmid pSIP609 and helpful discussions, and Dr. Lukas Mach for advice on protein expression and helpful discussions.

\section{Compliance with ethical standards}

Conflict of interest The authors declare that they have no conflict of interest in the publication.

\section{References}

Adler B, de la Peña Moctezuma A (2010) Leptospira and leptospirosis. Vet Microbiol 140:287-296. https://doi.org/10.1016/j.vetmi c.2009.03.012

Branger $C$ et al (2001) Identification of the hemolysis-associated protein 1 as a cross-protective immunogen of Leptospira interrogans by adenovirus-mediated vaccination. Infect Immun 69:68316838. https://doi.org/10.1128/iai.69.11.6831-6838.2001

Brown RA, Blumerman S, Gay C, Bolin C, Duby R, Baldwin CL (2003) Comparison of three different leptospiral vaccines for induction of a type 1 immune response to Leptospira borgpetersenii serovar Hardjo. Vaccine 21:4448-4458. https://doi. org/10.1016/s0264-410x(03)00439-0

Cortes-Perez NG, Lefèvre F, Corthier G, Adel-Patient K, Langella P, Bermúdez-Humarán LG (2007) Influence of the route of immunization and the nature of the bacterial vector on immunogenicity of mucosal vaccines based on lactic acid bacteria. Vaccine 25:6581-6588. https://doi.org/10.1016/j.vaccine.2007.06.062

de Vries MC, Vaughan EE, Kleerebezem M, de Vos WM (2006) Lactobacillus plantarum - survival, functional and potential probiotic properties in the human intestinal tract. Int Dairy J 16:1018-1028. https://doi.org/10.1016/j.idairyj.2005.09.003

del Rio B, Dattwyler RJ, Aroso M, Neves V, Meirelles L, Seegers JF, Gomes-Solecki M (2008) Oral immunization with recombinant Lactobacillus plantarum induces a protective immune response in mice with Lyme disease. Clin Vaccine Immunol 15:1429-1435. https://doi.org/10.1128/CVI.00169-08

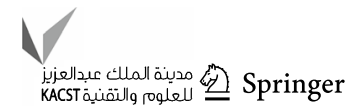


del Rio B, Seegers JF, Gomes-Solecki M (2010) Immune response to Lactobacillus plantarum expressing Borrelia burgdorferi OspA is modulated by the lipid modification of the antigen. PLoS One 5:e11199. https://doi.org/10.1371/journal.pone.0011199

Deveson Lucas DS, Cullen PA, Lo M, Srikram A, Sermswan RW, Adler B (2011) Recombinant LipL32 and LigA from leptospira are unable to stimulate protective immunity against leptospirosis in the hamster model. Vaccine 29:3413-3418. https://doi. org/10.1016/j.vaccine.2011.02.084

Diep DB, Mathiesen G, Eijsink VGH, Nes IF (2009) Use of lactobacilli and their pheromone-based regulatory mechanism in gene expression and drug delivery. Curr Pharm Biotechnol 10:62-73

Enkhbayar P, Kamiya M, Osaki M, Matsumoto T, Matsushima N (2004) Structural principles of leucine-rich repeat (LRR) proteins. Proteins 54:394-403. https://doi.org/10.1002/prot.10605

Fredriksen L, Mathiesen G, Sioud M, Eijsink VG (2010) Cell wall anchoring of the 37-kilodalton oncofetal antigen by Lactobacillus plantarum for mucosal cancer vaccine delivery. Appl Environ Microbiol 76:7359-7362. https://doi.org/10.1128/aem.01031-10

Grangette C, Müller-Alouf H, Geoffroy M, Goudercourt D, Turneer M, Mercenier A (2002) Protection against tetanus toxin after intragastric administration of two recombinant lactic acid bacteria: impact of strain viability and in vivo persistence. Vaccine 20:3304-3309

Haake DA, Mazel MK, McCoy AM, Milward F, Chao G, Matsunaga J, Wagar EA (1999) Leptospiral outer membrane proteins OmpL1 and LipL41 exhibit synergistic immunoprotection. Infect Immun 67:6572-6582

Harlow E, Lane D (1988) Antibodies: a laboratory manual, 1st edn. Cold Spring Harbor Laboratory Press, USA

Hniman A, Prapong S (2007) Development of leptospira molecular markers by using bioinformation from predicted leucine-rich repeat (LRR) protein genes. J Thai Vet Med Assoc 58:65-78

Ho SN, Hunt HD, Horton RM, Pullen JK, Pease LR (1989) Sitedirected mutagenesis by overlap extension using the polymerase chain reaction. Gene 77:51-59

Josson K et al (1989) Characterization of a gram-positive broad-hostrange plasmid isolated from Lactobacillus hilgardii. Plasmid 21:9-20

Kim YG et al (2006) Probiotic Lactobacillus casei activates innate immunity via NF-kappaB and p38 MAP kinase signaling pathways. Microbes Infect 8:994-1005. https://doi.org/10.1016/j.micin f.2005.10.019

Kleerebezem M et al (2003) Complete genome sequence of Lactobacillus plantarum WCFS1. Proc Natl Acad Sci USA 100:1990-1995. https://doi.org/10.1073/pnas.0337704100

Kobe B, Kajava AV (2001) The leucine-rich repeat as a protein recognition motif. Curr Opin Struct Biol 11:725-732. https://doi. org/10.1016/s0959-440x(01)00266-4

Maassen CB et al (1999) Instruments for oral disease-intervention strategies: recombinant Lactobacillus case $i$ expressing tetanus toxin fragment $\mathrm{C}$ for vaccination or myelin proteins for oral tolerance induction in multiple sclerosis. Vaccine 17:2117-2128

Mathiesen G, Sveen A, Piard JC, Axelsson L, Eijsink VG (2008) Heterologous protein secretion by Lactobacillus plantarum using homologous signal peptides. J Appl Microbiol 105:215-226. https ://doi.org/10.1111/j.1365-2672.2008.03734.x

Mathiesen G, Sveen A, Brurberg MB, Fredriksen L, Axelsson L, Eijsink VG (2009) Genome-wide analysis of signal peptide functionality in Lactobacillus plantarum WCFS1. BMC Genomics 10:425. https://doi.org/10.1186/1471-2164-10-425

Medaglini D, Pozzi G, King TP, Fischetti VA (1995) Mucosal and systemic immune responses to a recombinant protein expressed on the surface of the oral commensal bacterium Streptococcus gordonii after oral colonization. Proc Natl Acad Sci USA 92:6868-6872

Mohamadzadeh M, Duong T, Sandwick SJ, Hoover T, Klaenhammer TR (2009) Dendritic cell targeting of Bacillus anthracis protective antigen expressed by Lactobacillus acidophilus protects mice from lethal challenge. Proc Natl Acad Sci USA 106:4331-4336. https://doi.org/10.1073/pnas.0900029106

Nguyen TT et al (2011a) A food-grade system for inducible gene expression in Lactobacillus plantarum using an alanine racemaseencoding selection marker. J Agric Food Chem 59:5617-5624. https://doi.org/10.1021/jf104755r

Nguyen TT et al (2011b) Quantitative transcript analysis of the inducible expression system pSIP: comparison of the overexpression of Lactobacillus spp. beta-galactosidases in Lactobacillus plantarum. Microb Cell Fact 10:46. https://doi.org/10.1186/1475-2859-10-46

Nitipan S (2013) Molecular cloning and sequence analysis of the genes encoding leucine-rich repeat proteins of pathogenic Leptospira borgpetersenii. Doctoral thesis. Kasetsart University, Thailand

Nitipan S, Sritrakul T, Kunjantarachot A, Prapong S (2013) Identification of epitopes in Leptospira borgpetersenii leucine-rich repeat proteins. Infect Genet Evol 14:46-57. https://doi.org/10.1016/j. meegid.2012.10.014

Obst M, Meding ER, Vogel RF, Hammes WP (1995) Two genes encoding the beta-galactosidase of Lactobacillus sake. Microbiology 141:3059-3066

Palaniappan RUM, McDonough SP, Divers TJ, Chen C-S, Pan M-J, Matsumoto M, Chang Y-F (2006) Immunoprotection of recombinant leptospiral immunoglobulin-like protein A against Leptospira interrogans serovar pomona infection. Infect Immun 74:17451750. https://doi.org/10.1128/iai.74.3.1745-1750.2006

Roche FM et al (2003) Characterization of novel LPXTG-containing proteins of Staphylococcus aureus identified from genome sequences. Microbiology 149:643-654. https://doi.org/10.1099/ mic.0.25996-0

Seepersaud R, Hanniffy SB, Mayne P, Sizer P, Le Page R, Wells JM (2005) Characterization of a novel leucine-rich repeat protein antigen from group B streptococci that elicits protective immunity. Infect Immun 73:1671-1683. https://doi.org/10.1128/ iai.73.3.1671-1683.2005

Shaw DM et al (2000) Engineering the microflora to vaccinate the mucosa: serum immunoglobulin $\mathrm{G}$ responses and activated draining cervical lymph nodes following mucosal application of tetanus toxin fragment $\mathrm{C}$-expressing lactobacilli. Immunology 100:510-518

Shimosato $\mathrm{T}$ et al (2005) Augmentation of $\mathrm{T}(\mathrm{H})-1$ type response by immunoactive AT oligonucleotide from lactic acid bacteria via toll-like receptor 9 signaling. Biochem Biophys Res Commun 326:782-787. https://doi.org/10.1016/j.bbrc.2004.11.119

Silva ÉF et al (2007) The terminal portion of leptospiral immunoglobulin-like protein LigA confers protective immunity against lethal infection in the hamster model of leptospirosis. Vaccine 25:6277-6286. https://doi.org/10.1016/j.vaccine.2007.05.053

Sørvig E, Grönqvist S, Naterstad K, Mathiesen G, Eijsink VG, Axelsson L (2003) Construction of vectors for inducible gene expression in Lactobacillus sakei and L plantarum. FEMS Microbiol Lett 229:119-126

Sørvig E, Mathiesen G, Naterstad K, Eijsink VGH, Axelsson L (2005) High-level, inducible gene expression in Lactobacillus sakei and Lactobacillus plantarum using versatile expression vectors. Microbiology 151:2439-2449. https://doi.org/10.1099/ mic.0.28084-0

Trimpin S, Brizzard B (2009) Analysis of insoluble proteins. Biotechniques 46:409-419. https://doi.org/10.2144/000113168

Yigang XU, Yijing LI (2008) Construction of recombinant Lactobacillus casei efficiently surface displayed and secreted porcine parvovirus VP2 protein and comparison of the immune responses induced by oral immunization. Immunology 124:68-75. https:// doi.org/10.1111/j.1365-2567.2007.02738.x 